IIIIIIIIIIIIIIIIIIIIIIIIIIIIIIIIIIII

Original Article

IIIIIIIIIIIIIIIIIIIIIIIIIIIIIIIIIII

\title{
Activation of cell proliferation in Arabidopsis root meristem by isoprothiolane
}

\author{
Miyuki Kusajıma, Mai Inoue, Moeka Fujita, Kodai Miyagawa, \\ Rina Horita and Hideo NAKASHITA* \\ Research Center for Bioresources Development, Faculty of Biotechnology, Fukui Prefectural University, \\ Awara 910-4103, Japan
}

(Received June 27, 2018; Accepted August 23, 2018)

\begin{abstract}
A plant growth regulating agent "Fuji-one" has been used to control non-parasitic damping-off (Murenae disease) of rice seedlings. Its active ingredient, isoprothiolane (diisopropyl 1,3-dithiolan-2-ylidenemalonate, IPT), enhances root elongation of rice and Arabidopsis. To understand the mechanisms of IPT's effect on root development, its effect on Arabidopsis root cells was investigated histologically. IPT at a lower concentration $(12.5 \mu \mathrm{g} / \mathrm{mL})$ had no effect on root cell elongation, whereas it enhanced cell division in the root meristem. Histological analysis using phytohormone-related mutants indicated that jasmonic acid and ethylene were involved in the enhanced cell division. In contrast, IPT at a higher concentration $(75 \mu \mathrm{g} / \mathrm{mL})$ suppressed both cell elongation and cell division, in which jasmonic acid and ethylene were not involved. In addition, root hair formation was suppressed by treatment with IPT. These analyses demonstrated that IPT $(12.5 \mu \mathrm{g} / \mathrm{mL})$ enhanced root elongation by activating cell division in a jasmonic acid- and ethylene-dependent manner. ๑ Pesticide Science Society of Japan

Keywords: isoprothiolane, root meristem, ethylene, jasmonic acid, Arabidopsis.
\end{abstract}

\section{Introduction}

Isoprothiolane (diisopropyl 1,3-dithiolan-2-ylidenemalonate, IPT) is an active ingredient of a rice blast controlling agent " $\mathrm{Fu}-$ ji-one" (Nihon Nohyaku Co., Ltd.) ${ }^{1,2)}$ and has been used also as a plant growth regulating agent to protect rice seedlings cultured in nursery flats from non-parasitic damping-off (Murenae disease) (Fig. 1). ${ }^{3)}$ A plausible mechanism for IPT's plant growth regulating activity is acceleration of rice root development, which was observed at IPT concentrations as low as $6 \mu \mathrm{g} / \mathrm{mL}$, however, the molecular mechanism remains to be clarified. ${ }^{4,5)}$ Extensive studies on IPT's plant growth regulating activity in rice have demonstrated various physiological effects on rice; the promotion of auxin activity in lamina inclination assay, ${ }^{6}$ the induction of ethylene (ET) production in the seedlings and callus, ${ }^{6}$ the enhancement of acid phosphatase activity in the root, ${ }^{7)}$ the upregulation of methionine sulfoxide reduction leading to ET production, ${ }^{8)}$ and the promotion of water permeability of the root cell membrane. ${ }^{5)}$ However, without genetic studies in rice, the contribution of these physiological changes to enhancement of root development cannot been determined.

Recently, a more detailed analysis using Arabidopsis indicated

\footnotetext{
* To whom correspondence should be addressed.

E-mail: nakashita@fpu.ac.jp

Published online October 28, 2018

(c) Pesticide Science Society of Japan
}

that IPT possesses dual activities for the enhancement and suppression of primary root elongation at a lower concentration $(12.5 \mu \mathrm{g} / \mathrm{mL})$ and at a higher concentration $(75 \mu \mathrm{g} / \mathrm{mL})$, respectively, in Arabidopsis as well as in rice. $\left.{ }^{9}\right)$ Furthermore, analyses using phytohormone-related Arabidopsis mutants and phytohormone biosynthesis inhibitors demonstrated that auxin-, jasmonic acid (JA)- and ET-mediated signals are involved in the positive effect of a lower IPT concentration. ${ }^{9)}$ During plant growth under normal conditions, endogenous auxin is known to function in root elongation by activating cell division in the root apical meristem, ${ }^{10}$ ) whereas JA and ET, important phytohormones functioning in stress responses, are known to suppress root elongation. ${ }^{11,12)}$ Although the roles of these phytohormones in the plant growth regulating activity of IPT are important, the question has been raised as to how JA and ET function in root elongation. Thus, the present investigation was conducted to clarify the histological effect of IPT on Arabidopsis root growth in relation to JA and ET signals. Here we report the character-

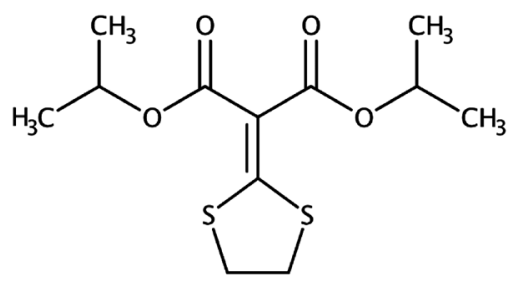

Fig. 1. Structure of isoprothiolane (IPT). 
ization of IPT's effects on the cell length, cell division, and formation of root hairs in the primary root.

\section{Materials and Methods}

\section{Plant growth conditions}

Surface-sterilized Arabidopsis seeds were placed on 1/2 Murashige and Skoog (MS) (Duchefa Biochemie) agar plates (containing vitamins, $1 \%$ sucrose, and $0.8 \%$ agar) $(140 \times 100 \times 14.5 \mathrm{~mm})$ and incubated in a vertical position under a constitutive light condition and a temperature of $22^{\circ} \mathrm{C}$ for 2 days. Seedlings were transferred to fresh $1 / 2 \mathrm{MS}$ agar plates or 1/2MS agar plates supplemented with IPT $(12.5$ or $75 \mu \mathrm{g} / \mathrm{mL})$ and incubated in a vertical position under the same condition.

\section{Cell length measurement}

Microscopic analysis of cell length in differentiation zone was performed with 7-day-old seedlings using a light microscope BX51 (Olympus) with an UPLanFLN 20X (NA 0.50) lens (Olympus) and a DP73 digital camera (Olympus). To compare the cell length between treatments, the trichoblast cell that shifted from the elongation step to the differentiation step was chosen as reliable estimate of the actual extent of cell elongation. ${ }^{13)}$ From the images taken with the microscope system, the length of the first epidermal cell with a visible root hair bulge was measured using cellSens Standard 1.6 software (Olympus). The cell lengths of at least 20 different roots were measured for comparison and statistical analysis between treatments.

\section{Quantitative analysis of root hairs}

Root hairs in 7-day-old seedlings were viewed using a stereo microscope SZX12 (Olympus) with a DF PLAPO 1.2x PF (NA 0.11 ) lens (Olympus) and photographed using a DP73 digital camera (Olympus). The length of root hairs formed in a section between 1 to $6 \mathrm{~mm}$ from the first visible root hair bulge was measured using cellSens Standard 1.6 software (Olympus). Density was determined as the number of root hairs in the same section under a microscope. Data were taken from at least 4 different roots in each experiment.

\section{Acetocarmine staining}

Roots of 7-day-old seedlings were cut and incubated in acetocarmine solution, acc. to Kultschitzky (Tokyo Kasei) for $30 \mathrm{~min}$ at $78^{\circ} \mathrm{C}$, followed by incubation in $0.24 \mathrm{~N} \mathrm{HCl}$ in $20 \%$ methanol for $15 \mathrm{~min}$ at $57^{\circ} \mathrm{C}$. The root samples were washed with $40 \%, 20 \%$, and $10 \%$ ethanol for $15 \mathrm{~min}$ each at room temperature and then stored in $25 \%$ glycerol containing $5 \%$ ethanol till analysis. Stained root samples were viewed using a light microscope BX51 (Olympus) with an UPLanFLN 10X (NA 0.30) lens (Olympus) and photographed using a DP73 digital camera (Olympus). The stained meristem length was measured using cellSens Standard 1.6 software. Data were taken from at least 20 different roots in each experiment.

\section{Propidium iodide staining}

Roots of 5-day-old seedlings, 3 days after the IPT treatment, were cut and stained with $10 \mu \mathrm{g} / \mathrm{mL}$ propidium iodide (Nacalai) for $2 \mathrm{~min}$, followed by rinsing in water to remove excess staining solution. Fluorescence in roots was detected using an LSM510 confocal laser scanning microscope (Zeiss) with a PlanAPOCHROMAT 20X objective lens (NA 0.8) (Zeiss). Propidium iodide was viewed at excitation wavelength of $543 \mathrm{~nm}$ and the fluorescence emission was collected between 608 and $629 \mathrm{~nm}$. Cells of the root cortex layer were counted in a file from the quiescent center to the first elongated cell. Cell numbers from at least 6 different roots were counted for comparison and statistical analysis between treatments.

\section{Results}

\section{Effect of IPT on cell elongation in Arabidopsis root}

We have previously reported that IPT has dual activities on root elongation in Arabidopsis, namely the enhancement of primary root elongation at a lower concentration $(12.5 \mu \mathrm{g} / \mathrm{mL})$ and suppression at a higher concentration $(75 \mu \mathrm{g} / \mathrm{mL}) .{ }^{9)}$ Considering the root cell development through three morphologically distinguishable zones, the cell division zone in meristem, the elongation zone, and the differentiation zone, IPT probably influences cell proliferation or cell elongation in the root. To determine IPT's effect on the primary root elongation, we first examined whether IPT influences the length of trichoblast cells in the lower part of the differentiation zone. After the end of elongation process, cell differentiation begins where the trichoblast cell forms a root hair. ${ }^{13,14)}$ Microscopic analysis indicated that length of the first epidermal cell with a visible root hair bulge was not influenced by $12.5 \mu \mathrm{g} / \mathrm{mL}$ IPT but was significantly reduced by $75 \mu \mathrm{g} / \mathrm{mL}$ IPT (Fig. 2A, B). These results suggest that the negative effect of IPT on root elongation is due to the reduction in cell length, however, the positive effect is not due to the increase in cell size.

In a previous study, analyses using mutants and biosynthetic inhibitors revealed that JA and ET are involved in IPT's positive effect on root elongation but not in its negative effect. ${ }^{9)}$ To determine the involvement of these phytohormones in IPT's effect on cell elongation, cell length was analyzed in jar1 and ein 2 mutants defective in JA- and ET-signal transduction, respectively. Microscopic analysis of jarl and ein 2 mutants exhibited results similar to those for the wild-type (Fig. 2B). These results suggest that JA and ET are not involved in the suppressive effect of IPT on cell elongation process at a higher concentration.

2. Effect of IPT on root hair formation in the differentiation zone Microscopic analysis showed that not only the cell length of the primary root but also root hair formation was affected by IPT treatment. To determine IPT's influence on root hair formation, we analyzed the length and density of root hairs in the primary root formed during IPT treatment. In the differentiation zone of the wild-type root, root hair length was reduced by treatment with $12.5 \mu \mathrm{g} / \mathrm{mL}$ IPT but was reduced more extremely 
when treated with $75 \mu \mathrm{g} / \mathrm{mL}$ (Fig. 3A, B). The root hair density in the differentiation zone was not affected by treatment with $12.5 \mu \mathrm{g} / \mathrm{mL}$ IPT but was significantly reduced by treatment with $75 \mu \mathrm{g} / \mathrm{mL}$ IPT (Fig. 3B). Thus, the higher concentration of IPT has negative effects on both primary root growth and root hair formation. The lower concentration of IPT $(12.5 \mu \mathrm{g} / \mathrm{mL})$ has a negative effect on root hair elongation, whereas primary root elongation was enhanced by this concentration of IPT via JA and ET signal transduction. For a better understanding of the effect of $12.5 \mu \mathrm{g} / \mathrm{mL}$ IPT on root hair formation, the root hair length was analyzed in some phytohormone-related mutants. The reduction in root hair length by $12.5 \mu \mathrm{g} / \mathrm{mL}$ IPT treatment was also observed in jar1, ein2, salicylic acid (SA) biosynthesisdeficient mutant sid2, and a NahG transgenic plant expressing an SA-degrading enzyme, as well as in the wild-type (Fig. 3C, D), which indicated that JA, ET, and SA were not involved in the reduction in root hair length by IPT.

A

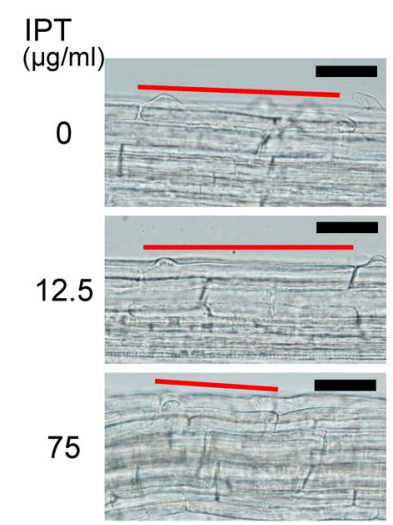

B

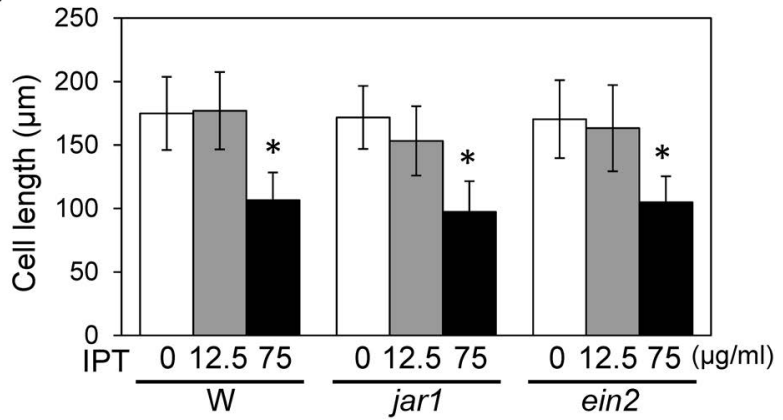

Fig. 2. Effect of IPT on cell length in differentiation zone. Seedlings were placed on $1 / 2 \mathrm{MS}$ medium or $1 / 2 \mathrm{MS}$ medium supplemented with 12.5 or $75 \mu \mathrm{g} / \mathrm{mL}$ IPT and cultured for 5 days. (A) Cell length in the differentiation zones of wild-type seedlings. The length of the first epidermal cell with a visible root hair bulge (marked with thin red lines) was measured. Thick bars $=50 \mu \mathrm{m}$. (B) Length of the first epidermal cell with a visible root hair bulge of the wild-type, jar1, and ein 2 seedlings. Data shown are the average and standard deviation (SD) $(n=45)$. Asterisks indicate statistically significant differences between non-treated control and IPTtreated seedlings (unpaired $t$ test, $p<0.05$ ). The experiment was repeated three times with similar results.
A

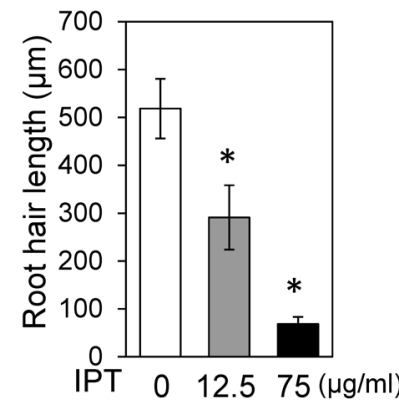

$\mathrm{B}$

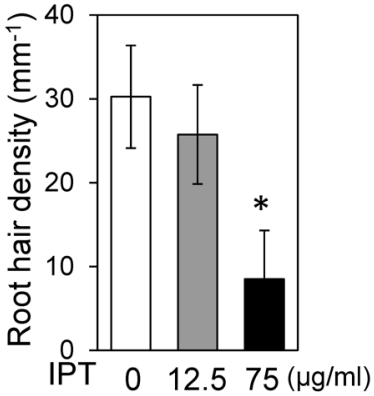

C

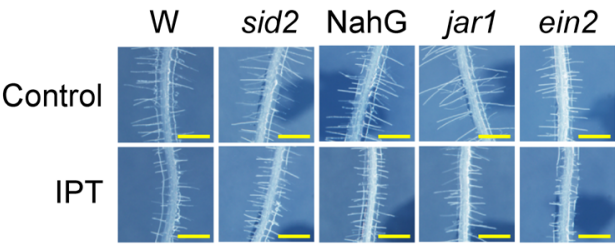

$\mathrm{D}$

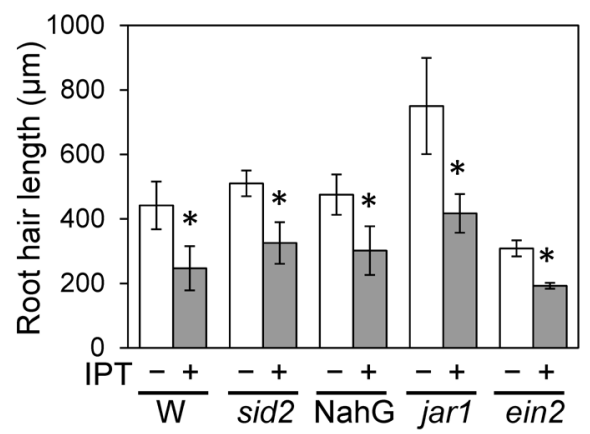

Fig. 3. Effect of IPT on root hair formation. Seedlings were placed on $1 / 2 \mathrm{MS}$ medium or $1 / 2 \mathrm{MS}$ medium supplemented with 12.5 or $75 \mu \mathrm{g} / \mathrm{mL}$ IPT and cultured for 5 days. (A) Length of root hairs formed in the differentiation zones of wild-type seedlings. Data shown are the average and SD $(n=40)$. Asterisks indicate statistically significant differences between non-treated control and IPT-treated seedlings in each genotype (unpaired $t$ test, $p<0.05)$. The experiment was repeated three times with similar results. (B) Root hair densities in the differentiation zones of the wild-type seedlings. Data shown are the average and SD $(n=6)$. Asterisks indicate statistically significant differences between non-treated control and IPTtreated seedlings (unpaired $t$ test, $p<0.05$ ). The experiment was repeated three times with similar results. (C) Photographs of root hairs formed in the zones of indicated genotypes. Non-treated control and $12.5 \mu \mathrm{g} / \mathrm{mL}$ IPT-treated seedlings are shown. Bars $=500 \mu \mathrm{m}$. (D) Length of root hairs formed in the differentiation zones of indicated genotypes. Data shown are the average and SD $(n=40)$. Asterisks indicate statistically significant differences between non-treated control and IPT-treated seedlings in each genotype (unpaired $t$ test, $p<0.05$ ). The experiment was repeated three times with similar results.

\section{Effect of IPT on meristem size}

Treatment with $12.5 \mu \mathrm{g} / \mathrm{mL}$ IPT enhanced the primary root elongation, whereas the cell length in the differentiation zone was not affected. This fact suggested the possibility that the positive effect of IPT on primary root elongation is due to enhanced cell proliferation in the meristem. To examine this possibility, the root meristem sizes of IPT-treated seedlings were compared 
with those of non-treated control seedlings. ${ }^{15)}$ Stereo microscopic analysis of acetocarmine-stained roots indicated that the meristem size of root treated with $12.5 \mu \mathrm{g} / \mathrm{mL}$ IPT was significantly larger than that of non-treated control (Fig. 4A, B). This result revealed that the meristem size was enlarged by treatment with $12.5 \mu \mathrm{g} / \mathrm{mL}$ IPT, probably due to increased cell proliferation in the meristem. In contrast, the meristem size was reduced by treatment with $75 \mu \mathrm{g} / \mathrm{mL}$ (Fig. 4A, B), indicating that cell proliferation in the meristem was suppressed by a higher concentration of IPT.

\section{Roles of phytohormones in enhanced cell proliferation by IPT} To understand the detailed mechanism of the increase in meristem size, the effect of $12.5 \mu \mathrm{g} / \mathrm{mL}$ IPT on the cell number in the meristem was examined via confocal microscopic analysis of the propidium iodide-stained root. ${ }^{16)}$ The cell number of the wildtype root treated with $12.5 \mu \mathrm{g} / \mathrm{mL}$ IPT was $c a .17 \%$ higher than that of non-treated control root, indicating that IPT activated the cell division in the meristem (Fig. 5A, B). The increase in cell number by IPT treatment was not observed in the root of jar1 nor ein 2 mutants, indicating that the activation of cell division

A

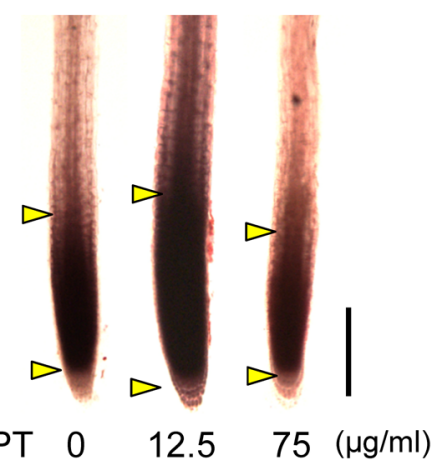

$\mathrm{B}$

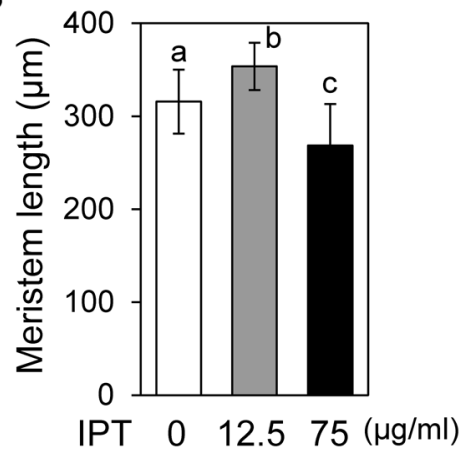

Fig. 4. Effect of IPT on meristem size. Seedlings were placed on a $1 / 2 \mathrm{MS}$ medium or $1 / 2 \mathrm{MS}$ medium supplemented with $12.5 \mathrm{or} 75 \mu \mathrm{g} / \mathrm{mL}$ IPT and cultured for 5 days. Roots were cut from the seedlings and used for acetocarmine staining. (A) Photographs of acetocarimine-stained meristems. Arrows indicate the start and end of the meristems. Bar $=200 \mu \mathrm{m}$. (B) Meristem size of non-treated control and IPT-treated seedlings. Data shown are the average and SD $(n=20)$. Different letters indicate statistically significant differences between treatments (ANOVA, $p<0.05$ ). The experiment was repeated two times with similar results.

\section{A}

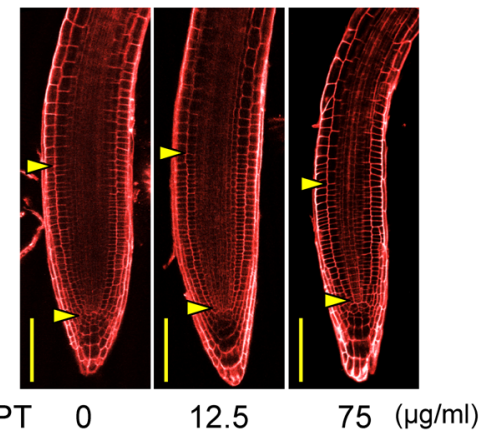

B

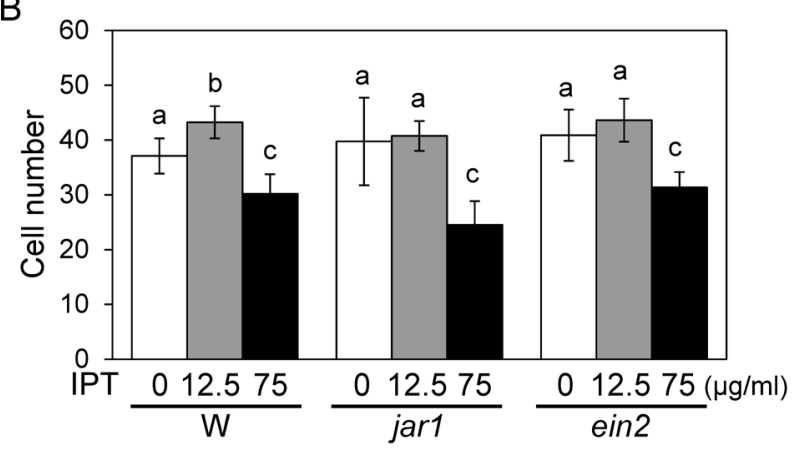

Fig. 5. Effect of IPT on cell proliferation in cell division zone. Seedlings were placed on a $1 / 2 \mathrm{MS}$ medium or $1 / 2 \mathrm{MS}$ medium supplemented with 12.5 or $75 \mu \mathrm{g} / \mathrm{mL}$ IPT and cultured for 3 days. Roots were cut from the seedlings and used for propidium iodide staining. (A) Photographs of propidium iodide-stained wild-type roots. Arrows indicate the quiescent center and the end of the cell division zone. Bars $=100 \mu \mathrm{m}$. (B) Cell number of the cell division zones in non-treated control and IPT-treated roots of indicated genotypes. Data shown are the average and SD $(n=6)$. Different letters indicate statistically significant differences between treatments in each genotype (ANOVA, $p<0.05$ ). The experiment was repeated three times with similar results.

by IPT treatment was dependent on JA and ET signals (Fig. 5B). Taken together with the cell length in the differentiation zone, this indicates that the lower concentration of IPT enhances primary root elongation by activating cell division in the meristem in a JA- and ET-dependent manner but not by enhancing the elongation process in elongation and differentiation zones.

On the other hand, the higher concentration of IPT $(75 \mu \mathrm{g} /$ $\mathrm{mL}$ ) reduced the cell number compared to that of non-treated plants, which result was also observed in jar1 and ein 2 mutants (Fig. 5A, B). Taken together, the negative effect of $75 \mu \mathrm{g} / \mathrm{mL}$ IPT on primary root elongation resulted from suppression of both cell elongation and cell division.

\section{Discussion}

Our results demonstrate that the positive effect of $12.5 \mu \mathrm{g} / \mathrm{mL}$ IPT on primary root elongation in Arabidopsis is due to the JA- and ET-signal-dependent increased cell proliferation in the meristem but not due to changes of cell elongation. In contrast, a higher concentration of IPT $(75 \mu \mathrm{g} / \mathrm{mL})$ suppresses both cell division and cell elongation of primary root. In addition, the 
presented data demonstrate that IPT affects root hair formation, where root hair length was more sensitive to IPT than was its density. These data indicate that IPT $(75 \mu \mathrm{g} / \mathrm{mL})$ has negative effects on cell division, cell elongation, and root hair formation in primary root development, all of which are independent from JA and ET signals. In contrast, IPT's positive effect on cell proliferation in the meristem is dependent on JA and ET.

In the cell division zone, IPT exhibited dual activities of promoting and suppressing cell proliferation, which should contribute to the enhancement and suppression of primary root elongation, respectively. Considering the necessity of JA and ET for IPT's positive effect on cell proliferation, each of the dual activities is regulated independently of each other. On the other hand, for the cell elongation process from the end of the cell division zone to the differentiation zone via the elongation zone, IPT exhibited only the negative effect to suppress cell elongation, which should contribute to the suppression of primary root growth by a higher concentration of IPT. Whether these negative effects on cell proliferation and cell elongation are based on the same action of IPT is unknown, because no molecule and signal transduction in these activities have been identified.

Our previous study demonstrated that the intermediate concentration of IPT $(37.5 \mu \mathrm{g} / \mathrm{mL})$ had no effect on primary root growth in Arabidopsis, probably because the independently regulated positive and negative effects neutralized each other at this concentration. ${ }^{9)}$ Based on the results presented here, at least three types of effects, increased and reduced cell division and suppression of cell elongation, should counterbalance one another. We have attempted to confirm the balanced effects histologically at this concentration, however, we have not yet succeeded because visible effects in the meristem and differentiation zone are faint under this condition.

Although JA and ET are known to suppress root growth, we previously reported, based on analyses using phytohormonerelated mutants and inhibitors, that JA and ET take part in IPT's positive effect on primary root elongation; this was confirmed as increased cell proliferation by histological analysis as shown here. ${ }^{9)}$ Recently, it was reported that JA and ET promote cell division in the quiescent center of the root apical meristem; however, in those studies, these hormones function negatively in root elongation. ${ }^{17,18)}$ Since cell division in the root growth process is intricately regulated by internal and external conditions in the root apical meristem that consists of many types of cells, its detailed regulation mechanisms remain to be clarified. Our finding offers an important clue to identifying the regulation mechanism of cell proliferation in the root apical meristem.

\section{Acknowledgements}

We thank Nihon Nohyaku Co., Ltd. for providing IPT. We also thank H. Takeuchi, A. Konishi, N. Kamikyo, and R. Komatsuzawa for technical assistances.

\section{References}

1) F. Araki and Y. Miyagi: Jpn. J. Phytopathol. 42, 401-406 (1976).

2) K. Kakiki and T. Misato: J. Pestic. Sci. 4, 305-313 (1979).

3) M. Hikawa and I. Yanai: Shokubutsu no Kagakuchosetsu 18, 71 (1983) (in Japanese).

4) T. Otsuka and H. Saka: Jpn. J. Crop. Sci. 56, 571-576 (1987), in Japanese.

5) M. Yoshida and M. Yukimoto: J. Pestic. Sci. 18, 385-387 (1993).

6) T. Otsuka and H. Saka: Jpn. J. Crop. Sci. 57, 631-635 (1988), in Japanese.

7) T. Otsuka and H. Saka: Jpn. J. Crop. Sci. 57, 722-727 (1988), in Japanese.

8) M. Uchida, M. Kanauchi and T. Sugimoto: Agric. Biol. Chem. 49, 1131-1135 (1985).

9) M. Kusajima, M. Nagata, N. Miyashita, Y. Yotagakiuchi, K. Maehara, I. Miyazaki, M. Inoue, M. Fujita and H. Nakashita: J. Pestic. Sci., in press.

10) A. P. Mahonen, K. Tusscher, R. Siligato, O. Smetana, S. Diaz-Trivino, J. Salojavi, G. Wachsman, K. Prasad, R. Heidstra and B. Sheres: Nature 515, 125-129 (2014).

11) Y. Zhang and J. G. Tumer: PLoS One 3, e3699 (2008).

12) B. Ma, C. C. Yin, S. J. He, X. Lu, W. K. Zhang, T. G. Lu, S. Y. Chen and J. S. Zhang: PLoS Genet. 10, e1004701 (2014).

13) T. De Cnodder, K. Vissenberg, D. Van Der Straeten and J.-P. Verbelen: New Phytol. 168, 541-550 (2005).

14) A. Bruex, R. M. Kainkaryam, Y. Wieckowski, Y. H. Kang, C. Bernhardt, Y. Xia, X. Zheng, J. Y. Wang, M. M. Lee, P. Benfey, P. J. Woolf and J. Schiefelbein: PLoS Genet. 8, e1002446 (2012).

15) J. Wopereis, E. Pajuelo, F. B. Dazzo, Q. Jiang, P. M. Gresshoff, F. J. De Bruijn, J. Stougaard and K. Szczyglowski: Plant J. 23, 97-114 (2000).

16) I. H. Street, S. Aman, Y. Zubo, A. Ramzan, X. Wang, S. N. Shakeel, J. J. Kieber and G. E. Schaller: Plant Physiol. 169, 338-350 (2015).

17) O. Ortega-Martinez, M. Pernas, R. J. Carol and L. Dolan: Science 317, 507-510 (2007).

18) Q. Chen, J. Sun, Q. Zhai, W. Zhou, L. Qi, L. Xu, B. Wang, R. Chen, H. Jiang, J. Qi, X. Li, K. Palme and C. Li: Plant Cell 23, 3335-3352 (2011). 